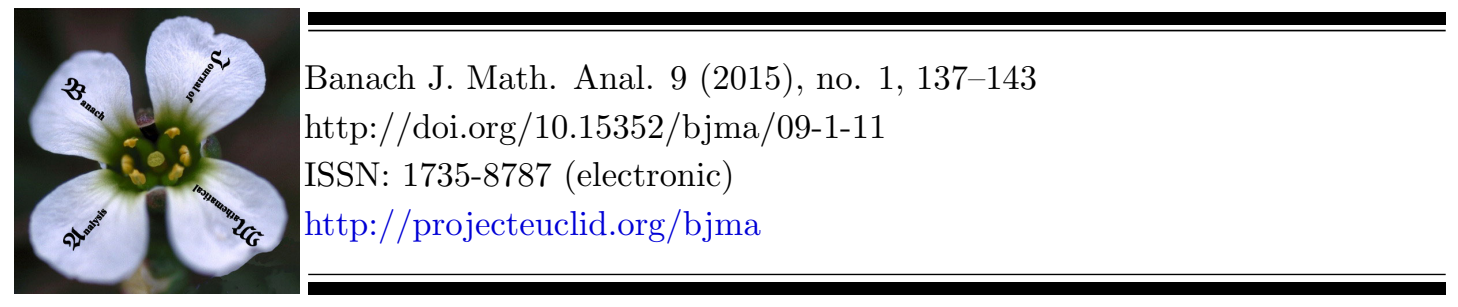

\title{
MULTIPLIERS IN PERFECT LOCALLY $m$-CONVEX ALGEBRAS
}

\author{
MARINA HARALAMPIDOU ${ }^{1 *}$, LOURDES PALACIOS ${ }^{2}$ AND CARLOS SIGNORET ${ }^{2}$ \\ Communicated by M. Abel
}

\begin{abstract}
In this paper we describe the multiplier algebra of a perfect complete locally $m$-convex algebra with an approximate identity and with complete Arens-Michael normed factors.
\end{abstract}

\section{Introduction And Preliminaries}

Multipliers are important in various areas of mathematics where an algebra structure appears (see e.g [1]; for (non-normed) topological algebras cf. e.g. [4]).

The algebras considered throughout are taken over the field of complexes $\mathbb{C}$. Denote by $L(E)$ the algebra of all linear operators on an algebra $E$.

Definition 1.1. A mapping $T: E \rightarrow E$ is called a left (right) multiplier on $E$ if $T(x y)=T(x) y(\operatorname{resp} . T(x y)=x T(y))$ for all $x, y \in E$; it is called a two-sided multiplier on $E$ if it is both a left and a right multiplier.

It is known that if $E$ is a proper algebra, namely $x E=\{0\}$ implies $x=0$ or $E x=\{0\}$ implies $x=0$, where 0 denotes the zero element of $E$, then any two-sided multiplier on $E$ is automatically a linear mapping [6, p. 20].

In the sequel, a two-sided multiplier will be called in short, a multiplier. We denote by $M_{l}(E)$ the set of all left multipliers on $E$, by $M_{r}(E)$ the set of all right multipliers on $E$ and by $M(E)$ that of all multipliers on $E$. Note that, by definition, $M(E)=M_{l}(E) \cap M_{r}(E)$.

Date: Received: Mar. 1, 2014; Revised: Apr. 1, 2014; Accepted: Apr. 10, 2014.

* Corresponding author.

2010 Mathematics Subject Classification. Primary 46H05; Secondary 46H10, 46K05.

Key words and phrases. Proper algebra, Arens-Michael decomposition, multiplier algebra, perfect projective system, perfect locally $m$-convex algebra. 
Obviously $M(E)$ is a subalgebra of $L(E)$ in case the algebra is proper. The same holds for $M_{r}(E)$ and $M_{l}(E)$. Now, for $x \in E$, the operator $l_{x}$ on $E$ given by $l_{x}(y)=x y, y \in E$, is, due to the associativity of $E$, a left multiplier. Similarly, we can also define the right multiplier with respect to $x \in E$, say $r_{x}$.

It is known that if $E$ is a proper algebra, then the mapping

$$
L: E \rightarrow M_{l}(E) \text { given by } x \mapsto l_{x}
$$

defines an algebra monomorphism which identifies $E$ with a subalgebra of $M_{l}(E)$. Moreover, $E$ is a left ideal of the algebra $M_{l}(E)$. A similar result is also valid for right multipliers. For multipliers, the algebra $E$ can be identified with a two-sided ideal in $M(E)$ ([3, p. 1933, Proposition 2.2 and p. 1934, Corollary 2.3]).

Definition 1.2. An approximate identity in a topological algebra $E$ is a net $\left(e_{\delta}\right)_{\delta \in \Delta}$ such that for each $x \in E$ we have

$$
\left(x-x e_{\delta}\right) \underset{\delta}{\rightarrow} 0 \text { and }\left(x-e_{\delta} x\right) \underset{\delta}{\rightarrow} 0 \text { for all } x \in E .
$$

Note that an algebra with an approximate identity is proper. In this paper we describe the multiplier algebra $M(E)$ in the case where $E$ is a certain complete locally $m$-convex algebra with an approximate identity.

For the sake of completeness, we recall what we mean by the "Arens-Michael decomposition" ([7, p. 88, Theorem 3.1]).

Let $\left(E,\left(p_{\alpha}\right)_{\alpha \in \Lambda}\right)$ be a complete locally $m$-convex algebra and

$$
\rho_{\alpha}: E \rightarrow E / \operatorname{ker}\left(p_{\alpha}\right) \equiv E_{\alpha} \text { defined by } \rho_{\alpha}(x)=x+\operatorname{ker}\left(p_{\alpha}\right) \equiv x_{\alpha}, \alpha \in \Lambda
$$

the respective quotient maps. Then $\dot{p}_{\alpha}\left(x_{\alpha}\right):=p_{\alpha}(x), x \in E, \alpha \in \Lambda$ defines on $E_{\alpha}$ an algebra norm, so that $E_{\alpha}$ is a normed algebra and the morphisms $\rho_{\alpha}, \alpha \in \Lambda$

are continuous. $\tilde{E}_{\alpha}, \alpha \in \Lambda$ denotes the completion of $E_{\alpha}$ (with respect to $\dot{p}_{\alpha}$ ). $\Lambda$ is endowed with a partial order by putting $\alpha \leq \beta$ if and only if $p_{\alpha}(x) \leq p_{\beta}(x)$ for every $x \in E$. Thus, $\operatorname{ker}\left(p_{\beta}\right) \subseteq \operatorname{ker}\left(p_{\alpha}\right)$ and hence the continuous (onto) morphism $f_{\alpha \beta}: E_{\beta} \rightarrow E_{\alpha}: x_{\beta} \mapsto f_{\alpha \beta}\left(x_{\beta}\right)=x_{\alpha}, \alpha \leq \beta$ is defined. Moreover, $f_{\alpha \beta}$ is extended to a continuous morphism $\bar{f}_{\alpha \beta}: \tilde{E}_{\beta} \rightarrow \tilde{E}_{\alpha}, \alpha \leq \beta$. Thus, $\left(E_{\alpha}, f_{\alpha \beta}\right),\left(\tilde{E}_{\alpha}, \bar{f}_{\alpha \beta}\right)$, $\alpha, \beta \in \Lambda$ with $\alpha \leq \beta$ are projective systems of normed (resp. Banach) algebras, so that $E \cong \lim E_{\alpha} \cong \lim \tilde{E}_{\alpha}$ (Arens-Michael decomposition) within topological algebra isomorphisms.

In [3, p. 1934, Theorem 3.1], it is shown that, in a special case, the algebra $M(E)$ is a subalgebra of $\mathcal{L}(E)$, the algebra of all continuous linear operators on $E$; for completeness, we refer it here.

Theorem 1.3. Let $\left(E,\left(p_{\alpha}\right)_{\alpha \in \Lambda}\right)$ be a complete locally $m$-convex algebra with an approximate identity $\left(e_{\delta}\right)_{\delta \in \Delta}$. Suppose that each factor $E_{\alpha}=E / \operatorname{ker} p_{\alpha}$ in the Arens-Michael decomposition of $E$ is complete. Then each multiplier $T$ of $E$ is continuous, viz. $M(E)$ is a subalgebra of $\mathcal{L}(E)$.

\section{Perfectness and Multipliers in locally $m$-Convex Algebras}

To proceed, we use the notion of a perfect projective system as it appeared in [2, p. 199, Definition 2.7]. To fix notation, we repeat it. 
Definition 2.1. A projective system $\left\{\left(E_{\alpha}, f_{\alpha \beta}\right)\right\}_{\alpha \in \Lambda}$ of topological algebras is called perfect, if the restrictions to the projective limit algebra

$$
E=\lim _{\longleftarrow} E_{\alpha}=\left\{\left(x_{\alpha}\right) \in \prod_{\alpha \in \Lambda} E_{\alpha}: f_{\alpha \beta}\left(x_{\beta}\right)=x_{\alpha}, \text { if } \alpha \leq \beta \in \Lambda\right\}
$$

of the canonical projections $\pi_{\alpha}: \prod_{\alpha \in \Lambda} E_{\alpha} \rightarrow E_{\alpha}, \alpha \in \Lambda$, namely, the (continuous algebra) morphisms

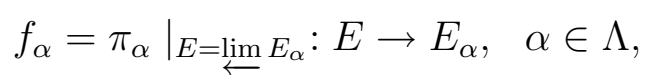

are onto maps. The resulting projective limit algebra $E=\underset{\lim }{\longleftarrow} E_{\alpha}$ is then called a perfect (topological) algebra.

Definition 2.2. In the sequel, by the term perfect locally $m$-convex algebra we mean a locally $m$-convex algebra $\left(E,\left(p_{\alpha}\right)_{\alpha \in \Lambda}\right)$ for which the respective ArensMichael projective system $\left\{\left(E_{\alpha}, f_{\alpha \beta}\right)\right\}_{\alpha \in \Lambda}$ is perfect.

Every Fréchet locally m-convex algebra $\left(E,\left(p_{n}\right)_{n \in \mathbb{N}}\right)$ gives a perfect projective system of normed algebras, and thus it is a perfect algebra (see [2], and [5]).

Example 2.3. Let $E$ be a non-complete normed algebra. Take $E=E_{\alpha}$ for each $\alpha \in \Lambda$ and, for $\alpha \leq \beta$, let $f_{\alpha \beta}: E_{\beta} \rightarrow E_{\alpha}$ be the identity map. Then $\Delta=$ $\lim _{\longleftarrow} E_{\alpha}$, the diagonal algebra, is a perfect locally $m$-convex algebra, but $\Delta$ is not complete.

Let $E=\left(E,\left(p_{\alpha}\right)_{\alpha \in \Lambda}\right)$ be a perfect complete locally $m$-convex algebra with an approximate identity and such that each factor $E_{\alpha}$ of its Arens-Michael decomposition is complete.

Remark 2.4. If $\phi$ is the isomorphism $E \longrightarrow \lim E_{\alpha}$ given by $\phi(x)=\left(x_{\alpha}\right)_{\alpha \in \Lambda}$, then, for each $\alpha \in \Lambda, \rho_{\alpha}=f_{\alpha} \circ \phi$. Therefore, $\operatorname{ker} p_{\alpha}=\operatorname{ker} \rho_{\alpha}=\operatorname{ker}\left(f_{\alpha} \circ \phi\right)$.

Remark 2.5. By the hypothesis of perfectness, each $f_{\beta}$ is surjective, so each time we have an element $x_{\beta} \in E_{\beta}$, we can choose an element $\omega \in E$ such that $\omega_{\beta}=x_{\beta}$, and consequently $\omega_{\alpha}=f_{\alpha \beta}\left(x_{\beta}\right)=x_{\alpha}$, whenever $\alpha \leq \beta$.

For each $\alpha \leq \beta$, we define the map $h_{\alpha \beta}: M\left(E_{\beta}\right) \rightarrow M\left(E_{\alpha}\right)$ given by

$$
\left[h_{\alpha \beta}\left(T_{\beta}\right)\right]\left(x_{\alpha}\right)=f_{\alpha \beta}\left(T_{\beta}\left(x_{\beta}\right)\right)
$$

which is well defined, according to the following lemma.

Lemma 2.6. Let $\left(E,\left(p_{\alpha}\right)_{\alpha \in \Lambda}\right)$ be a perfect complete locally $m$-convex algebra with an approximate identity $\left(e_{\delta}\right)_{\delta \in \Delta}$ and such that each factor $E_{\alpha}$ of its Arens-Michael decomposition is complete. Then $\operatorname{ker} f_{\alpha \beta}$ is $T_{\beta}$-invariant for each $T_{\beta} \in M\left(E_{\beta}\right)$, that is, $T_{\beta}\left(\operatorname{ker} f_{\alpha \beta}\right) \subseteq \operatorname{ker} f_{\alpha \beta}$, if $\alpha \leq \beta$, and the map $h_{\alpha \beta}$ is a well-defined continuous multiplicative linear mapping.

Proof. Take $x_{\beta} \in \operatorname{ker} f_{\alpha \beta}$. Since $E$ has an approximate identity $\left(e_{\delta}\right)_{\delta \in \Delta}$ and multipliers over Banach algebras are continuous (see [6, p. 20, Theorem 1.1.1]), then

$$
f_{\alpha \beta}\left(T_{\beta}\left(x_{\beta}\right)\right)=f_{\alpha \beta}\left(T_{\beta}\left(\lim _{\delta} x_{\beta} e_{\delta}\right)\right)=f_{\alpha \beta}\left(\lim _{\delta} T_{\beta}\left(x_{\beta} e_{\delta}\right)\right)=\lim _{\delta} f_{\alpha \beta}\left(T_{\beta}\left(x_{\beta} e_{\delta}\right)\right)=
$$




$$
=\lim _{\delta} f_{\alpha \beta}\left(x_{\beta} T_{\beta}\left(e_{\delta}\right)\right)=\lim _{\delta}\left[f_{\alpha \beta}\left(x_{\beta}\right) f_{\alpha \beta}\left(T_{\beta}\left(e_{\delta}\right)\right)\right]=0 .
$$

We claim that $h_{\alpha \beta}\left(T_{\beta}\right)$ is well-defined. For that, let $\alpha \leq \beta, x \in E$ be such that $x_{\alpha}=x_{\alpha}^{\prime}$ and $T_{\beta} \in M\left(E_{\beta}\right)$; then $0=x_{\alpha}-x_{\alpha}^{\prime}=\rho_{\alpha}(x)-\rho_{\alpha}\left(x^{\prime}\right)=\rho_{\alpha}\left(x-x^{\prime}\right)$ and hence $0=\left(f_{\alpha} \circ \phi\right)\left(x-x^{\prime}\right)=\left(f_{\alpha \beta} \circ f_{\beta} \circ \phi\right)\left(x-x^{\prime}\right)$, which implies that $\left(f_{\beta} \circ \phi\right)\left(x-x^{\prime}\right) \in \operatorname{ker} f_{\alpha \beta}$. Since ker $f_{\alpha \beta}$ is $T_{\beta}$-invariant, $T_{\beta}\left(\left(f_{\beta} \circ \phi\right)\left(x-x^{\prime}\right)\right) \in \operatorname{ker} f_{\alpha \beta}$ too, and therefore

$$
\begin{gathered}
0=f_{\alpha \beta}\left(T_{\beta}\left(\left(f_{\beta} \circ \phi\right)\left(x-x^{\prime}\right)\right)=f_{\alpha \beta}\left(T_{\beta}\left(\rho_{\beta}\left(x-x^{\prime}\right)\right)\right)=f_{\alpha \beta}\left(T_{\beta}\left(x_{\beta}-x_{\beta}^{\prime}\right)\right)=\right. \\
=f_{\alpha \beta}\left(T_{\beta}\left(x_{\beta}\right)\right)-f_{\alpha \beta}\left(T_{\beta}\left(x_{\beta}^{\prime}\right)\right),
\end{gathered}
$$

that is, $f_{\alpha \beta}\left(T_{\beta}\left(x_{\beta}\right)\right)=f_{\alpha \beta}\left(T_{\beta}\left(x_{\beta}^{\prime}\right)\right)$. This proves the claim.

Moreover, $h_{\alpha \beta}\left(T_{\beta}\right)$ is actually a multiplier on $E_{\alpha}$. For, let $x_{\alpha}$ and $y_{\alpha}$ be two elements in $E_{\alpha}$. Then

$$
\left[h_{\alpha \beta}\left(T_{\beta}\right)\right]\left(x_{\alpha} y_{\alpha}\right)=f_{\alpha \beta}\left(T_{\beta}\left(x_{\beta} y_{\beta}\right)\right)=f_{\alpha \beta}\left(x_{\beta} T_{\beta}\left(y_{\beta}\right)\right)=f_{\alpha \beta}\left(x_{\beta}\right) f_{\alpha \beta}\left(T_{\beta}\left(y_{\beta}\right)\right)=
$$

$x_{\alpha}\left(f_{\alpha \beta}\left(T_{\beta}\left(y_{\beta}\right)\right)=x_{\alpha}\left[h_{a \beta}\left(T_{\beta}\right)\right]\left(y_{\alpha}\right)\right.$ and so, $h_{\alpha \beta}\left(T_{\beta}\right)$ is a right multiplier. In a similar way, one can prove that $h_{\alpha \beta}\left(T_{\beta}\right)$ is a left multiplier.

It is easily seen that $h_{\alpha \beta}$ is a linear mapping. Moreover, $h_{\alpha \beta}$ is multiplicative. For that, take $T_{\beta}, S_{\beta} \in M\left(E_{\beta}\right)$. We have

$$
\left[h_{\alpha \beta}\left(T_{\beta} \circ S_{\beta}\right)\right]\left(x_{\alpha}\right)=f_{\alpha \beta}\left(\left(T_{\beta} \circ S_{\beta}\right)\left(x_{\beta}\right)\right)=f_{\alpha \beta}\left(T_{\beta}\left(S_{\beta}\left(x_{\beta}\right)\right)\right) .
$$

On the other hand, since the system is perfect, we can choose $\omega \in E$ (equivalently $\left.\left(\omega_{\alpha}\right)_{\alpha \in \Lambda} \in \lim _{\alpha} E_{\alpha}\right)$ such that $f_{\alpha \beta}\left(S_{\beta}\left(x_{\beta}\right)\right)=\omega_{\alpha}$; note that $f_{\alpha \beta}\left(\omega_{\beta}\right)=\omega_{\alpha}$ too. Then $S_{\beta}\left(x_{\beta}\right)-\omega_{\beta} \in \operatorname{ker} f_{\alpha \beta}$. But, since ker $f_{\alpha \beta}$ is $T_{\beta}$-invariant, we have $T_{\beta}\left(S_{\beta}\left(x_{\beta}\right)-\right.$ $\left.\omega_{\beta}\right) \in \operatorname{ker} f_{\alpha \beta}$, and thus $f_{\alpha \beta}\left(T_{\beta}\left(S_{\beta}\left(x_{\beta}\right)\right)\right)=f_{\alpha \beta}\left(T_{\beta}\left(\omega_{\beta}\right)\right)$. Besides,

$$
\begin{gathered}
f_{\alpha \beta}\left(T_{\beta}\left(S_{\beta}\left(x_{\beta}\right)\right)\right)=f_{\alpha \beta}\left(T_{\beta}\left(\omega_{\beta}\right)\right)=h_{\alpha \beta}\left(T_{\beta}\right)\left(\omega_{\alpha}\right)=h_{\alpha \beta}\left(T_{\beta}\right)\left(f_{\alpha \beta}\left(S_{\beta}\left(x_{\beta}\right)\right)\right)= \\
=h_{\alpha \beta}\left(T_{\beta}\right)\left(\left(h_{\alpha \beta}\left(S_{\beta}\right)\right)\left(x_{\alpha}\right)\right)=\left(h_{\alpha \beta}\left(T_{\beta}\right) \circ h_{a \beta}\left(S_{\beta}\right)\right)\left(x_{\alpha}\right) .
\end{gathered}
$$

The last, in connection with (2.1) gives the multiplicativity of $h_{\alpha \beta}$.

Next, we prove that $h_{\alpha \beta}$ is continuous. Since $f_{\alpha \beta}: E_{\beta} \rightarrow E_{\alpha}$ is a continuous mapping between normed algebras, there exists a constant $K>0$ such that $\dot{p}_{\alpha}\left(f_{\alpha \beta}\left(y_{\beta}\right)\right) \leq K \dot{p}_{\beta}\left(y_{\beta}\right)$ for each $y_{\beta} \in E_{\beta}$. In particular,

$$
\dot{p}_{\alpha}\left(f_{\alpha \beta}\left(T_{\beta}\left(x_{\beta}\right)\right)\right) \leq K \dot{p}_{\beta}\left(T_{\beta}\left(x_{\beta}\right)\right) \text { for each } x_{\beta} \in E_{\beta} .
$$

Taking the supremum on the right hand of (2.2) and since $M\left(E_{\beta}\right)$ is a Banach algebra (see [6, p. 20, Theorem 1.1.1]), we get

$$
\dot{p}_{\alpha}\left(f_{\alpha \beta}\left(T_{\beta}\left(x_{\beta}\right)\right)\right) \leq K \dot{p}_{\beta}\left(T_{\beta}\left(x_{\beta}\right)\right) \leq K \sup _{\dot{p}_{\beta}\left(x_{\beta}\right) \leq 1}\left\{\dot{p}_{\beta}\left(T_{\beta}\left(x_{\beta}\right)\right)\right\} \leq K\left\|T_{\beta}\right\|_{\beta}
$$

for every $x_{\beta} \in E_{\beta}$ with $\dot{p}_{\beta}\left(x_{\beta}\right) \leq 1$, and where $\|\cdot\|_{\beta}$ is the norm in the multiplier algebra $M\left(E_{\beta}\right)$. Since $f_{\alpha \beta}\left(T_{\beta}\left(x_{\beta}\right)\right)=\left[h_{\alpha \beta}\left(T_{\beta}\right)\right]\left(x_{\alpha}\right)$ whenever $\alpha \leq \beta$ (hence $\left.\dot{p}_{\alpha}\left(x_{\alpha}\right) \leq \dot{p}_{\beta}\left(x_{\beta}\right)\right)$, then $\dot{p}_{\alpha}\left(\left[h_{\alpha \beta}\left(T_{\beta}\right)\right]\left(x_{\alpha}\right)\right) \leq K\left\|T_{\beta}\right\|_{\beta}$ for every $x_{\alpha} \in E_{\alpha}$ with $\dot{p}_{\alpha}\left(x_{\alpha}\right) \leq 1$ by (2.3). Taking now the supremum in this latter relation, we have $\sup \dot{p}_{\alpha}\left(\left[h_{\alpha \beta}\left(T_{\beta}\right)\right]\left(x_{\alpha}\right)\right) \leq K\left\|T_{\beta}\right\|_{\beta}$. Thus $\left\|h_{\alpha \beta}\left(T_{\beta}\right)\right\|_{\alpha} \leq K\left\|T_{\beta}\right\|_{\beta}$, namely, $\dot{p}_{\alpha}\left(x_{\alpha}\right) \leq 1$

each $h_{\alpha \beta}$ is continuous. 
So far, we have the family of topological algebras $M\left(E_{\alpha}\right)$ and the family of multiplicative continuous linear mappings $h_{\alpha \beta}: M\left(E_{\beta}\right) \rightarrow M\left(E_{\alpha}\right), \alpha \leq \beta$ in $\Lambda$. Actually, they form a projective system. In fact, if $\alpha \leq \beta \leq \gamma$, then $f_{\alpha \beta} \circ f_{\beta \gamma}=$ $f_{\alpha \gamma}$, and therefore

$$
\begin{aligned}
& {\left[h_{\alpha \gamma}\left(T_{\gamma}\right)\right]\left(x_{\alpha}\right)=f_{\alpha \gamma}\left(T_{\gamma}\left(x_{\gamma}\right)\right)=\left(f_{\alpha \beta} \circ f_{\beta \gamma}\right)\left(T_{\gamma}\left(x_{\gamma}\right)\right)=f_{\alpha \beta}\left(f_{\beta \gamma}\left(T_{\gamma}\left(x_{\gamma}\right)\right)=\right.} \\
& \quad=f_{\alpha \beta}\left(\left[h_{\beta \gamma}\left(T_{\gamma}\right)\right]\left(x_{\beta}\right)\right)=\left[h_{\alpha \beta}\left(h_{\beta \gamma}\left(T_{\gamma}\right)\right)\right]\left(x_{\alpha}\right)=\left[\left(h_{\alpha \beta} \circ h_{\beta \gamma}\right)\left(T_{\gamma}\right)\right]\left(x_{\alpha}\right)
\end{aligned}
$$

for each $x_{\alpha} \in E_{\alpha}$. That is, $h_{\alpha \gamma}\left(T_{\gamma}\right)=\left(h_{\alpha \beta} \circ h_{\beta \gamma}\right)\left(T_{\gamma}\right)$ for each $T_{\gamma} \in M\left(E_{\gamma}\right)$, which implies that $h_{\alpha \gamma}=h_{\alpha \beta} \circ h_{\beta \gamma}$; it is clear that $h_{\alpha \alpha}=I d_{M\left(E_{\alpha}\right)}$.

Thus, we have the projective system of Banach algebras $\left\{\left(M\left(E_{\alpha}\right), h_{\alpha \beta}\right)\right\}_{\alpha \in \Lambda}$ and we can take its inverse $\operatorname{limit}, \lim M\left(E_{\alpha}\right)$.

Now, we prove a lemma that will be useful in the sequel.

Lemma 2.7. Let $\left(E,\left(p_{\alpha}\right)_{\alpha \in \Lambda}\right)$ be a locally $m$-convex algebra with an approximate identity $\left(e_{\delta}\right)_{\delta \in \Delta}$ and let $T \in M(E)$. Then, for each $\alpha \in \Lambda$, $\operatorname{ker} p_{\alpha}$ is $T$-invariant; that is, $T\left(\operatorname{ker} p_{\alpha}\right) \subseteq \operatorname{ker} p_{\alpha}$.

Proof. Take $x \in \operatorname{ker} p_{a}$. Since the seminorms are continuous, for $\varepsilon>0$, there exists an index $\delta_{0} \in \Delta$ such that $p_{\alpha}\left(T(x)-T(x) e_{\delta}\right)<\varepsilon$ whenever $\delta \geq \delta_{0}$. We have

$$
\begin{gathered}
p_{\alpha}(T(x))=p_{\alpha}\left(T\left(x-x e_{\delta_{0}}+x e_{\delta_{0}}\right)\right)=p_{\alpha}\left(T(x)-T\left(x e_{\delta_{0}}\right)+T\left(x e_{\delta_{0}}\right)\right) \\
\leq p_{\alpha}\left(T(x)-T\left(x e_{\delta_{0}}\right)\right)+p_{\alpha}\left(T\left(x e_{\delta_{0}}\right)\right)=p_{\alpha}\left(T(x)-T(x) e_{\delta_{0}}\right)+p_{\alpha}\left(x T\left(e_{\delta_{0}}\right)\right) \\
\leq p_{\alpha}\left(T(x)-T(x) e_{\delta_{0}}\right)+p_{a}(x) p_{\alpha}\left(T\left(e_{\delta_{0}}\right)\right)<\varepsilon .
\end{gathered}
$$

Since this is true for an arbitrary $\varepsilon>0$, we conclude that $p_{\alpha}(T(x))=0$, that is, $T(x) \in \operatorname{ker} p_{\alpha}$.

Now we state our main Theorem.

Theorem 2.8. Let $\left(E,\left(p_{\alpha}\right)_{\alpha \in \Lambda}\right)$ be a complete locally $m$-convex algebra with an approximate identity $\left(e_{\delta}\right)_{\delta \in \Delta}$, such that the respective projective system is perfect and each factor $E_{\alpha}=E / \operatorname{ker} p_{a}$ in its Arens-Michael decomposition is complete. Then $M(E) \cong \lim M\left(E_{\alpha}\right)$ within a topological algebra isomorphism.

Proof. Take $T \in M(E)$. Due to Lemma 2.7, $T$ induces a well-defined map $T_{\alpha}: E_{\alpha} \rightarrow E_{\alpha}$ such that $T_{\alpha} \circ \rho_{\alpha}=\rho_{\alpha} \circ T$ for each $\alpha \in \Lambda$, that is, $T_{\alpha}\left(x_{\alpha}\right)=$ $T_{\alpha}\left(\rho_{\alpha}(x)\right)=\rho_{\alpha}(T(x))=T(x)_{\alpha}$ for each $x \in E$. Since for $x_{\alpha}, y_{\alpha} \in E_{\alpha}$,

$$
T_{\alpha}\left(x_{\alpha} y_{\alpha}\right)=\rho_{a}(T(x y))=\rho_{\alpha}(x T(y))=x_{\alpha} T(y)_{\alpha}=x_{\alpha} T_{\alpha}\left(y_{\alpha}\right),
$$

$T_{\alpha}$ is a right multiplier. In a similar way it can be shown that it is a left multiplier, as well.

Note also that $\left(T_{\alpha}\right)_{\alpha \in \Lambda}$ is an element of $\lim _{\longleftarrow} M\left(E_{\alpha}\right)$. Indeed, for $\alpha \leq \beta$ and $\rho_{\alpha}(x)=x_{\alpha} \in E_{\alpha}$, we have

$$
\begin{gathered}
{\left[h_{\alpha \beta}\left(T_{\beta}\right)\right]\left(\rho_{\alpha}(x)\right)=\left[h_{\alpha \beta}\left(T_{\beta}\right)\right]\left(x_{\alpha}\right)=f_{\alpha \beta}\left(T_{\beta}\left(\left(x_{\beta}\right)\right)=f_{\alpha \beta}\left(T_{\beta}\left(\rho_{\beta}(x)\right)=\right.\right.} \\
=f_{\alpha \beta}\left(\rho_{\beta}(T(x))=f_{\alpha \beta}\left(\left(f_{\beta} \circ \phi\right)(T(x))\right)=\left(\left(f_{\alpha \beta} \circ f_{\beta}\right) \circ \phi\right)(T(x))=\right. \\
=\left(f_{\alpha} \circ \phi\right)(T(x))=\rho_{\alpha}(T(x))=T_{\alpha}\left(\rho_{\alpha}(x)\right) .
\end{gathered}
$$

Therefore $h_{\alpha \beta}\left(T_{\beta}\right)=T_{\alpha}$ if $\alpha \leq \beta$. 
Now we define the map

$$
\Phi: M(E) \longrightarrow \lim _{\longleftarrow} M\left(E_{\alpha}\right) \text { by } \Phi(T)=\left(T_{\alpha}\right)_{\alpha \in \Lambda},
$$

which obviously is linear. Moreover, for $T, S \in M(E)$ and $x_{\alpha} \in E_{\alpha}$, we have

$$
\begin{gathered}
\rho_{\alpha}(\Phi(T \circ S))\left(x_{\alpha}\right)=(T \circ S)_{\alpha}\left(x_{\alpha}\right)=((T \circ S)(x))_{\alpha}=\left(T(S(x))_{\alpha}=T_{\alpha}\left(S(x)_{\alpha}\right)=\right. \\
=T_{\alpha}\left(S_{\alpha}\left(x_{\alpha}\right)\right)=\left(T_{\alpha} \circ S_{\alpha}\right)\left(x_{\alpha}\right),
\end{gathered}
$$

which implies that $(T \circ S)_{\alpha}=T_{\alpha} \circ S_{\alpha}$, and therefore $\Phi(T \circ S)=\Phi(T) \circ \Phi(S)$, namely, $\Phi$ is multiplicative.

Next, we show that $\Phi$ is one to one. For that, take $T, S \in M(E)$ such that $\left(T_{\alpha}\right)_{\alpha \in \Lambda}=\Phi(T)=\Phi(S)=\left(S_{\alpha}\right)_{\alpha \in \Lambda}$; then $T_{\alpha}=S_{\alpha}$ for each $x \in E$ and for each $\alpha \in \Lambda$. Therefore $\rho_{\alpha} \circ T=\rho_{\alpha} \circ S$ for each $\alpha \in \Lambda$; then $T=S$. Moreover, $\Phi$ is an onto map. Indeed, for $\left(W_{\alpha}\right)_{\alpha \in \Lambda} \in \lim _{\longleftarrow} M\left(E_{\alpha}\right)$ define the map

$$
W: E \rightarrow E \text { by } W(x)=\phi^{-1}\left(\left(W_{\alpha}\left(x_{\alpha}\right)\right)_{\alpha \in \Lambda}\right),
$$

which obviously is linear. Also

$$
\begin{gathered}
\left.W(x y)=\phi^{-1}\left(\left(W_{\alpha}(x y)_{\alpha}\right)_{\alpha \in \Lambda}\right)=\phi^{-1}\left(W_{\alpha}\left(x_{\alpha} y_{\alpha}\right)\right)_{\alpha \in \Lambda}\right)=\phi^{-1}\left(\left(x_{\alpha} W_{\alpha}\left(y_{\alpha}\right)\right)_{\alpha \in \Lambda}\right)= \\
=\phi^{-1}\left(\left(x_{\alpha}\right)_{\alpha \in \Lambda}\right) \phi^{-1}\left(\left(W_{\alpha}\left(y_{\alpha}\right)\right)_{\alpha \in \Lambda}\right)=x W(y)
\end{gathered}
$$

and similarly on the other side, so $W$ is a multiplier on $E$. Finally, it is clear that $\Phi(W)=\left(W_{\alpha}\right)_{\alpha \in \Lambda}$.

We claim that $\Phi$ is continuous. By [3, p. 1934, Theorem 3.1], $M(E)$ is a subalgebra of $\mathcal{L}(E)$, the algebra of all continuous linear operators on $E$, so that the topology on $M(E)$ is the operator topology. We denote by

$$
g_{\alpha}: M(E) \rightarrow M\left(E_{\alpha}\right)
$$

the map $g_{\alpha}(T)=T_{\alpha}$, which, by Lemma 2.7, is well defined and obviously linear. Let us denote by $h_{\alpha}: \lim _{\longleftarrow} M\left(E_{\alpha}\right) \rightarrow M\left(E_{\alpha}\right)$ the canonical continuous homomorphism from the inverse limit to one of its factors. Note that $h_{\alpha} \circ \phi=g_{\alpha}$ holds for each $\alpha \in \Lambda$.

Since $\Phi$ is continuous if and only if, for each $\alpha \in \Lambda, f_{\alpha} \circ \Phi$ is continuous (see [7, p. 89, the proof of Theorem 3.1]), we have to prove that $g_{\alpha}$ is continuous (for each $\alpha \in \Lambda$ ). We recall that the topology of $M(E)$ can be given by the set of seminorms $\left(\bar{p}_{\alpha}\right)_{\alpha \in \Lambda}$ defined as $\bar{p}_{\alpha}(T)=\sup _{p_{\alpha}(x) \leq 1} p_{\alpha}(T(x))$ for each $T \in M(E)$. Further, the topology of $M\left(E_{\alpha}\right)$ can be given by the norm $\|\cdot\|_{\alpha}$ defined as $\|S\|_{\alpha}=$ sup $\dot{p}_{\alpha}(S(x))$ for each $S \in M\left(E_{\alpha}\right)$, where, as usual, $\dot{p}_{\alpha}$ is the induced norm in $\dot{p}_{\alpha}(x) \leq 1$

$E_{\alpha}$ given by $\dot{p}_{\alpha}\left(x_{\alpha}\right)=\dot{p}_{\alpha}\left(x+\operatorname{ker} p_{\alpha}\right)=p_{\alpha}(x)$. The topology of $\lim _{\longleftarrow} M\left(E_{\alpha}\right)$ can be defined by the local base consisting of neighborhoods $V=\bigcap_{i=1}^{n} h_{\alpha_{i}}^{-1}\left(V_{\alpha_{i}}\right)$, where $V_{\alpha_{i}}$ is a basic neighborhood in $M\left(E_{\alpha_{i}}\right)$.

Let $\varepsilon_{i}>0$ be given and let

$$
V_{\alpha_{i}}=\left\{S \in M\left(E_{\alpha_{i}}\right):\|S\|_{\alpha_{i}}<\varepsilon_{i}\right\} \text { and } U_{\alpha_{i}}=\left\{T \in M(E): \bar{p}_{\alpha_{i}}(T)<\varepsilon_{i}\right\} .
$$

We claim that

$$
T \in U_{\alpha_{i}} \Longleftrightarrow T_{\alpha_{i}} \in V_{\alpha_{i}} .
$$


Indeed,

$$
\begin{gathered}
T \in U_{\alpha_{i}} \Longleftrightarrow \bar{p}_{\alpha_{i}}(T)<\varepsilon_{i} \Longleftrightarrow \sup _{p_{\alpha_{i}}(x) \leq 1} p_{\alpha_{i}}(T(x))<\varepsilon_{i} \\
\Longleftrightarrow \sup _{\dot{p}_{\alpha_{i}}\left(x_{\alpha_{i}}\right) \leq 1} \dot{p}_{\alpha_{i}}\left((T(x))_{\alpha_{i}}\right)<\varepsilon_{i} \Longleftrightarrow \sup _{\dot{p}_{\alpha_{i}}\left(x_{\alpha_{i}}\right) \leq 1} \dot{p}_{\alpha_{i}}\left(T_{\alpha_{i}}\left(x_{\alpha_{i}}\right)\right)<\varepsilon_{i} \\
\Longleftrightarrow\left\|T_{\alpha_{i}}\right\|_{\alpha_{i}}<\varepsilon_{i} \Longleftrightarrow T_{\alpha_{i}} \in V_{\alpha_{i}} .
\end{gathered}
$$

Now, let $V_{\alpha}$ be a basic neighborhood of 0 in $M\left(E_{\alpha}\right)$, say

$$
V_{\alpha}=\left\{S \in M\left(A_{\alpha}\right):\|S\|_{\alpha}<\varepsilon\right\} .
$$

Put $U_{\alpha}=g_{\alpha}^{-1}\left(V_{\alpha}\right)$. Then $U_{\alpha}=\left\{T \in M(E): \bar{p}_{\alpha}(T)<\varepsilon\right\}$. This implies the continuity of $g_{\alpha}$ for each $\alpha \in \Lambda$. Hence $\Phi$ is continuous.

Finally, we show that $\Phi$ is an open map. Let $V=\bigcap_{i=1}^{n} h_{\alpha_{i}}^{-1}\left(V_{\alpha_{i}}\right)$ be a basic neighborhood of 0 in $M(E)$. Take $T \in V$; then $T \in h_{\alpha_{i}}^{-1}\left(V_{\alpha_{i}}\right)$ for all $i=1, \ldots, n$. Therefore $h_{\alpha_{i}}(T) \in V_{\alpha_{i}}$ and, due to (2.4), $T_{\alpha_{i}} \in U_{\alpha_{i}}$. Then $\Phi(T) \in U=\left(U_{\alpha}\right)$, where $U_{\alpha}=U_{\alpha_{i}}$ for $\alpha=\alpha_{1}, \alpha_{2}, \ldots, \alpha_{n}$ and $U_{\alpha}=M\left(E_{\alpha}\right)$ otherwise. This proves that $\Phi$ is an open map, and the proof is complete.

Acknowledgements. We want to thank Professor Mohamed Oudadess for the suggestion of Example 2.3 and for his fruitful comments. We are especially indebted to the referee for his pertinent remarks.

\section{REFERENCES}

1. G.F. Bachelis and J.W. McCoy, Left centralizers of an $H^{*}$-algebra, Proc. Amer. Math. Soc. $\mathbf{4 3}(1)(1974), 106-110$.

2. M. Haralampidou, The Krull nature of locally $C^{*}$-algebras, Function Spaces (Edwardsville, IL, 2002), 195-200, Contemp. Math. 328, Amer. Math. Soc., Providence, RI, 2003.

3. M. Haralampidou, L. Palacios and C. Signoret, Multipliers in locally convex *-algebras, Rocky Mountain J. Math. 43, No. 6, 2013, 1931-1940.

4. T. Husain, Multipliers of topological algebras, Dissertationes Math. (Rozprawy Mat.) 285(1989), 40 pp.

5. G. Köthe, Topological Vector Spaces, I. Springer-Verlag, Berlin, 1969.

6. R. Larsen, The multiplier problem, Lectures Notes in Math. No. 105, Springer-Verlag, Berlin, 1969.

7. A. Mallios, Topological Algebras. Selected Topics, North-Holland, Amsterdam, 1986.

1 Department of Mathematics, University of Athens, Panepistimioupolis, Athens 15784, GREECE.

E-mail address: mharalam@math.uoa.gr

2 Universidad Autónoma Metropolitana Iztapalapa, Mexico City 09340, MexICO.

E-mail address: pafa@xanum.uam.mx

E-mail address: casi@xanum.uam.mx 